\title{
Review of bottomonium studies at Belle
}

\author{
Roman $\mathrm{Mizuk}^{1, *}$ \\ ${ }^{1}$ P.N. Lebedev Physical Institute of the Russian Academy of Sciences, Moscow 119991
}

\begin{abstract}
We review recent bottomonium studies at Belle. The results include the new measurement of the $\eta_{b}(1 S)$ mass, the observation of the $\Upsilon(4 S) \rightarrow$ $\Upsilon(1 S) \eta^{\prime}$ transition, and the observation of the $e^{+} e^{-} \rightarrow \chi_{b J}(1 P) \pi^{+} \pi^{-} \pi^{0}$ process in the $\Upsilon(11020)$ region.
\end{abstract}

\section{Introduction}

B-factories, the BaBar and Belle experiments, produced many highlights in the bottomonium physics. Among them are:

- observation of the spin-singlet states $\eta_{b}(1 S), \eta_{b}(2 S), h_{b}(1 P)$ and $h_{b}(2 P)$;

- observation of charged bottomonium-like states $Z_{b}(10610)$ and $Z_{b}(10650)$ with exotic quark content;

- observation of anomalous transitions from the $\Upsilon(4 S), \Upsilon(10860)$ and $\Upsilon(11020)$.

Here we present three recent results from Belle: the new measurement of the $\eta_{b}(1 S)$ mass [1], the observation of the $\Upsilon(4 S) \rightarrow \Upsilon(1 S) \eta^{\prime}$ transition [2], and the observation of the $e^{+} e^{-} \rightarrow$ $\chi_{b J}(1 P) \pi^{+} \pi^{-} \pi^{0}$ process in the $\Upsilon(11020)$ region [3].

\section{New measurement of the $\eta_{b}(1 S)$ mass}

There is a substantial disagreement between various measurements of the $\eta_{b}(1 S)$ mass [4]. Those from the $\Upsilon(2 S, 3 S) \rightarrow \eta_{b}(1 S) \gamma$ transitions measured by BaBar and CLEO are grouped near $9390 \mathrm{MeV} / c^{2}$, while those from the $h_{b}(1 P, 2 P) \rightarrow \eta_{b}(1 S) \gamma$ transitions measured by Belle are clustered near $9400 \mathrm{MeV} / c^{2}$. To improve on this, Belle studied the $\Upsilon(2 S) \rightarrow \eta_{b}(1 S) \gamma$ transition using the $24.7 \mathrm{fb}^{-1} \Upsilon(2 S)$ data sample.

Like previous measurements, Belle used inclusive reconstruction: the energy spectrum of all photons was investigated. Selection requirements include suppression of the light quark production using event topology and $\pi^{0}$ veto. The $\gamma$ spectrum after subtraction of the smooth component of the fit function is shown in figure 1. The significance of the $\eta_{b}(1 S)$ signal exceeds 7 standard deviations $(\sigma)$, and this is the first observation of the $\Upsilon(2 S) \rightarrow \eta_{b}(1 S) \gamma$ transition. The $\eta_{b}(1 S)$ width is fixed to the world-average value, and only the branching fraction and the mass are reported. For the branching fraction measurement, a non-relativistic Breit-Wigner (BW) signal shape is used, like in all previous measurements. The result $\mathcal{B}\left(\Upsilon(2 S) \rightarrow \eta_{b}(1 S) \gamma\right)=\left(6.1_{-0.7-0.6}^{+0.6+0.9}\right) \times 10^{-4}$ agrees with the world average [4]. For the mass measurement, the BW shape multiplied by the photon energy to the third power is used.

\footnotetext{
*e-mail: mizuk@lebedev.ru
} 


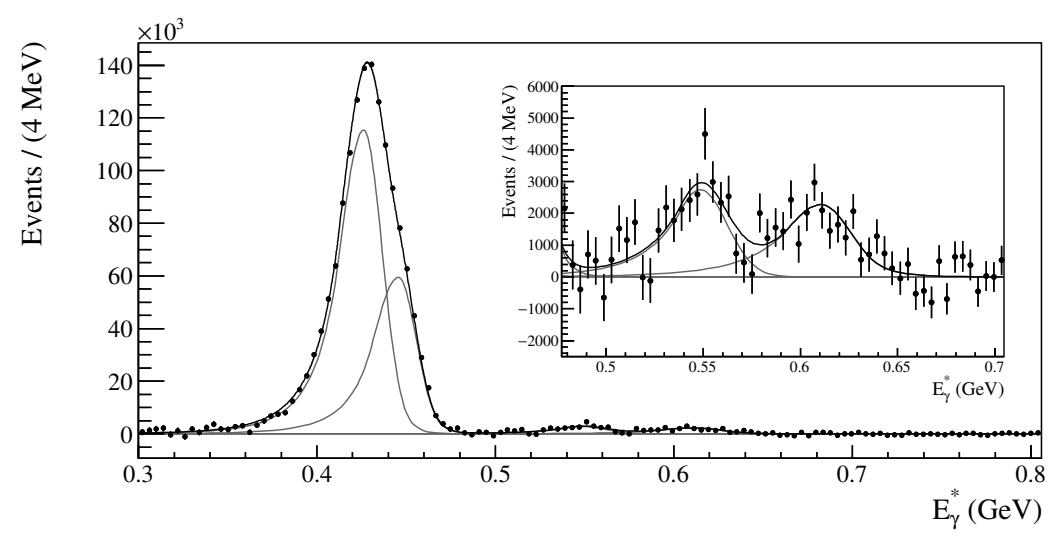

Figure 1. The inclusive photon spectrum after subtraction of the background component of the fit. The black curve indicates the fit to the data, and the gray curves indicate the individual signal components. The $\chi_{b 1,2}(1 P) \rightarrow \gamma \Upsilon(1 S)$ transitions at $\sim 424$ and $\sim 442 \mathrm{MeV}$ are dominant. The inset contains the same information with the scale chosen to highlight the ISR and $\eta_{b}(1 S)$ signal peaks, appearing at $\sim 550$ and $\sim 600 \mathrm{MeV}$, respectively.

The result $M\left(\eta_{b}(1 S)\right)=9394.8_{-3.1-2.7}^{+2.7+4.5} \mathrm{MeV} / c^{2}$ is just between the two groups of measurements mentioned above, consistent with both of them within the uncertainties. If the $E_{\gamma}^{3}$ term is not used, the mass shifts by $2.6 \mathrm{MeV} / c^{2}$ to higher values. We conclude that more precise measurement is needed to solve the puzzle of the $\eta_{b}(1 S)$ mass.

\section{Observation of the $\Upsilon(4 S) \rightarrow \Upsilon(1 S) \eta^{\prime}$ transition}

Both BaBar and Belle observed many decays of the $\Upsilon(4 S)$, $(10860)$ and $\Upsilon(11020)$ states that do not agree with the expectations for pure bottomonium states (for review see, e.g., [5]). Puzzling properties correspond to a violation of the OZI rule and Heavy Quark Spin Symmetry; their explanation might be a contribution of hadron loops or, equivalently, the $B$ hadron admixture in the $\Upsilon$ states. Recently, Belle reported the observation of a new transition, $\Upsilon(4 S) \rightarrow \Upsilon(1 S) \eta^{\prime}$, using the $496 \mathrm{fb}^{-1}$ data sample collected at the $\Upsilon(4 S)$.

The decay chains $\Upsilon(4 S) \rightarrow \Upsilon(1 S) \eta^{\prime}, \Upsilon(1 S) \rightarrow \mu^{+} \mu^{-}$or $e^{+} e^{-}, \eta^{\prime} \rightarrow \pi^{+} \pi^{-} \gamma$ or $\pi^{+} \pi^{-} \eta$, $\eta \rightarrow \gamma \gamma$ were used. The signals were identified using the $\Delta M=M(\Upsilon(4 S))-M(\Upsilon(1 S))-M\left(\eta^{\prime}\right)$ variable, where $M(X)$ is the mass of all the particles comprising the $X$ candidate. The distributions in the $\Delta M$ for the $\eta^{\prime} \rightarrow 2 \pi 1 \gamma$ and $2 \pi 2 \gamma$ final states are shown in figure 2. Each distribution shows a clear signal with a significance of about $4 \sigma$; the combined significance including systematic uncertainty is $5.7 \sigma$. The branching fraction is measured to be $\mathcal{B}\left(\Upsilon(4 S) \rightarrow \Upsilon(1 S) \eta^{\prime}\right)=(3.43 \pm 0.88 \pm 0.21) \times 10^{-5}$. Belle also reported the $\eta^{\prime}$ to $\eta$ production ratio of $0.20 \pm 0.06$, which agrees with the expectations of the $B$ hadron admixture model [6].

\section{Observation of $e^{+} e^{-} \rightarrow \chi_{b J}(1 P) \pi^{+} \pi^{-} \pi^{0}$ in the $\Upsilon(11020)$ region}

Among about ten known anomalous hadronic transitions from the $\Upsilon(10860)$ [5], the energy dependence was measured only for the $e^{+} e^{-} \rightarrow \Upsilon(n S) \pi^{+} \pi^{-}(n=1,2,3)$ [7] and $e^{+} e^{-} \rightarrow$ $h_{b}(n P) \pi^{+} \pi^{-}(n=1,2)$ [8]. Recently Belle studied also the $e^{+} e^{-} \rightarrow \chi_{b J}(1 P) \pi^{+} \pi^{-} \pi^{0}$ processes in the energy region above the $\Upsilon(10860)$ using scan data with about $1 \mathrm{fb}^{-1}$ per point. 

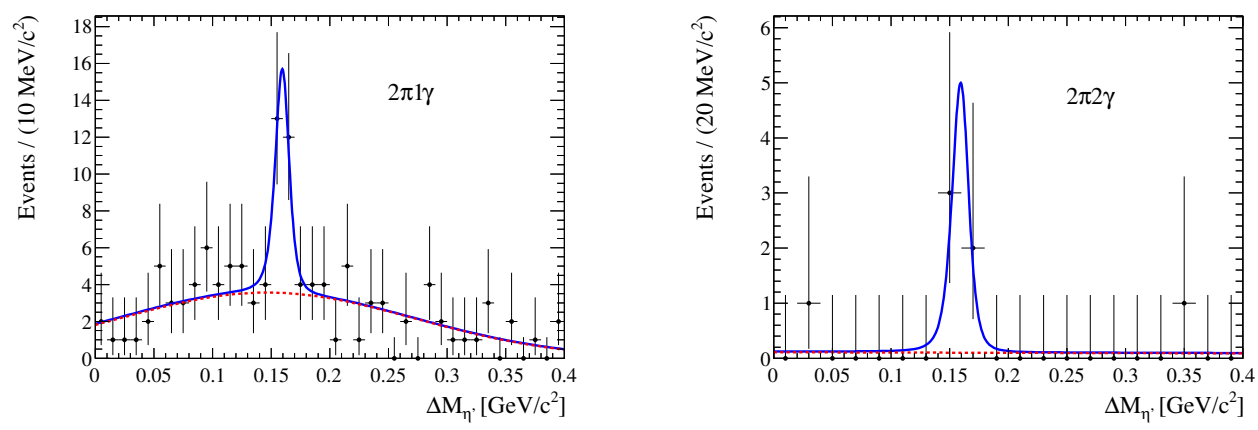

Figure 2. Fit to the $\Delta M_{\eta^{\prime}}$ distribution for $\Upsilon(4 S) \rightarrow \eta^{\prime} \Upsilon(1 S)$ candidates reconstructed in the $2 \pi 1 \gamma$ (left) and $2 \pi 2 \gamma$ (right) final states. Data are shown as points, the solid blue line shows the best fit to the data, while the dashed red line shows the background contribution.

Full reconstruction was used, with the $\chi_{b J}(1 P)$ reconstructed in the $\mu^{+} \mu^{-} \gamma$ final state. A scatter plot of $M\left(\pi^{+} \pi^{-} \pi^{0}\right)$ versus $M(\gamma \Upsilon(1 S))$ for six energy scan data samples near the $\Upsilon(11020)$ is shown in figure 3 . There is a clear clustering of events in the $\chi_{b J}(1 P)$ sig-
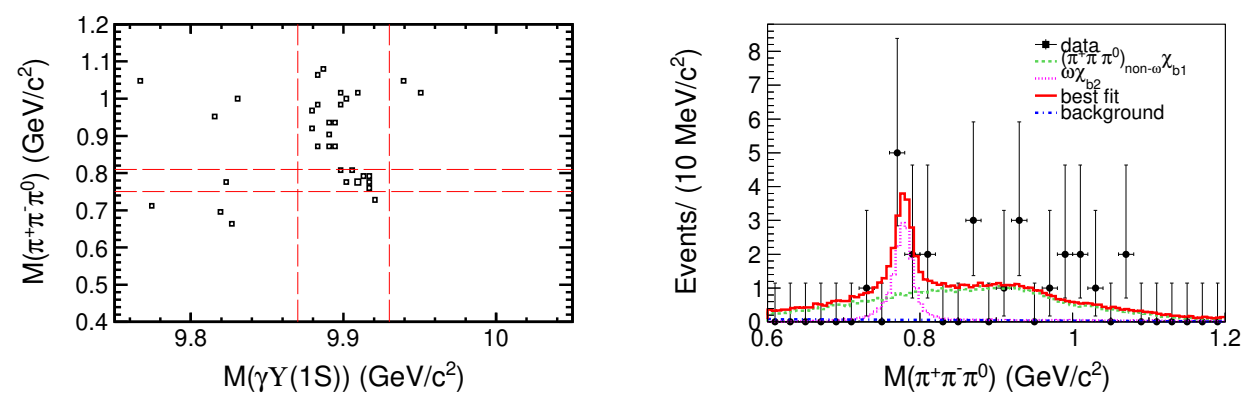

Figure 3. (Left) A scatter plot of $M\left(\pi^{+} \pi^{-} \pi^{0}\right)$ versus $M\left(\gamma^{\gamma} \Upsilon(1 S)\right)$ from data. Red dashed lines indicate signal regions of the $\chi_{b J}(1 P)$ and $\omega$. (Right) The projections of the $2 \mathrm{D}$ fit for events in the $\chi_{b J}$ signal region. Points with error bars are data, solid lines are the best fit, dashed lines are the $\chi_{b 1}$ signals, dotted lines are $\chi_{b 2}$ signals, and the dash-dotted lines are the fitted background.

nal region. Along the $M\left(\pi^{+} \pi^{-} \pi^{0}\right)$ axis, there is a cluster of events in the $\omega$ region and there are many events at higher masses. It is evident that there is a correlation between the $M\left(\pi^{+} \pi^{-} \pi^{0}\right)$ and $M\left(\gamma^{\Upsilon} \Upsilon(1 S)\right)$ variables: $\omega$ candidates are produced together with $\chi_{b 2}$, while higher mass $\pi^{+} \pi^{-} \pi^{0}$ combinations are accompanied by $\chi_{b 1}$. The significance of the $e^{+} e^{-} \rightarrow \chi_{b 1}(1 P)\left(\pi^{+} \pi^{-} \pi^{0}\right)_{\text {non- } \omega}$ signal is $5.3 \sigma$, thus it is the first observation of this process in the energy region of the $\Upsilon(11020)$. The significance of the $e^{+} e^{-} \rightarrow \chi_{b 2}(1 P) \omega$ is $4.0 \sigma$. These values are obtained from a $2 \mathrm{D}$ fit of the $M\left(\pi^{+} \pi^{-} \pi^{0}\right)$ versus $M\left(\gamma^{\top} \Upsilon(1 S)\right)$ distribution.

To estimate the energy dependence of the $e^{+} e^{-} \rightarrow \chi_{b J}(1 P) \pi^{+} \pi^{-} \pi^{0}$ cross section, the $2 \mathrm{D}$ fit is not repeated at every scan point. Instead, the number of signal events is counted in the combined $\chi_{b 1}$ and $\chi_{b 2}$ signal region and background is subtracted using sidebands. The events are not separated into the $\omega$ or higher $\pi^{+} \pi^{-} \pi^{0}$ mass samples. The resulting cross sections are presented in figure 4 . There are three points with very high accuracy; these are the 


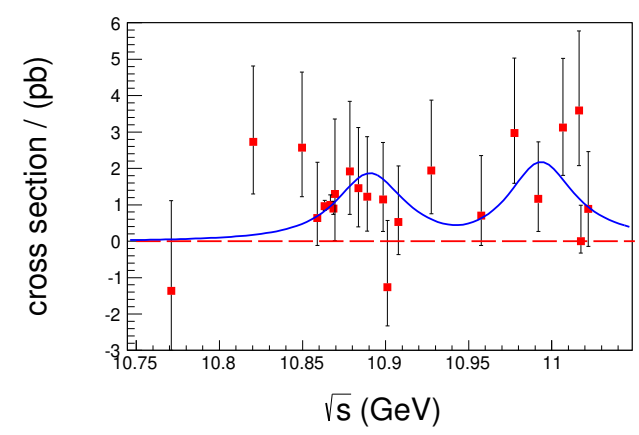

Figure 4. Fitting to the cross sections of $e^{+} e^{-} \rightarrow \pi^{+} \pi^{-} \pi^{0} \chi_{b J}$. Red boxes with error bars are the cross sections of $e^{+} e^{-} \rightarrow \pi^{+} \pi^{-} \pi^{0} \chi_{b J}$ and solid blue curve is fitting curve.

measurements in the $\Upsilon(10860)$ on-resonance data that were reported by Belle previously [9]. The accuracy is insufficient to conclude whether the production mechanism is resonant, nonresonant or both. Assuming that the mechanism is resonant, the cross sections are fitted using a sum of Breit-Wigner amplitudes to represent the $\Upsilon(10860)$ and $\Upsilon(11020)$ contributions. The fit results are presented in figure 4. Belle finds $\mathcal{B}\left(\Upsilon(10860) \rightarrow \chi_{b J}(1 P) \pi^{+} \pi^{-} \pi^{0}\right)=(2.5 \pm 0.6 \pm$ $2.0 \pm 0.7) \times 10^{-3}$ and $\mathcal{B}\left(\Upsilon(11020) \rightarrow \chi_{b J}(1 P) \pi^{+} \pi^{-} \pi^{0}\right)=\left(8.7 \pm 4.3 \pm 6.1_{-2.5}^{+4.5}\right) \times 10^{-3}$. These results agree with the expectations of the $B$ hadron loops model [10].

Belle searched also for the $e^{+} e^{-} \rightarrow \chi_{b J}(1 P) \phi$ processes in the $\Upsilon(11020)$ region using the data of six scan points and found no significant signal. These processes are expected to be strongly suppressed (factor $10^{3}$ ) compared to the $e^{+} e^{-} \rightarrow \chi_{b J}(1 P) \omega[10]$.

\section{Conclusions}

We presented here three recent Belle results on bottomonium. Among on-going analyses to be reported soon are the measurement of the energy dependence of the $e^{+} e^{-} \rightarrow B^{(*)} \bar{B}^{(*)}(\pi)$ cross sections, update of the $Z_{b}(10610)$ and $Z_{b}(10650)$ lineshapes in the $B \bar{B}$ and $B \bar{B}^{*}$ channels, search for the $W_{b J}$ states in the $\Upsilon(10860) \rightarrow W_{b J} \gamma \rightarrow\left(\Upsilon(1 S) \pi^{+} \pi^{-}\right) \gamma$ transitions, search for the $\Upsilon(4 S, 5 S) \rightarrow \eta_{b}(1 S, 2 S) \omega$ transitions and others.

Support from the Russian Foundation for Basic Research (Grant \# 17-02-00485) is gratefully acknowledged.

\section{References}

[1] B. G. Fulsom et al. [Belle Collaboration], Phys. Rev. Lett. 121, 232001 (2018).

[2] E. Guido et al. [Belle Collaboration], Phys. Rev. Lett. 121, 062001 (2018).

[3] J. H. Yin et al. [Belle Collaboration], Phys. Rev. D 98, 091102 (2018).

[4] M. Tanabashi et al. (Particle Data Group), Phys. Rev. D 98, 030001 (2018).

[5] A. E. Bondar, R. V. Mizuk and M. B. Voloshin, Mod. Phys. Lett. A 32, 1750025 (2017).

[6] M. B. Voloshin, Mod. Phys. Lett. A 26, 773 (2011).

[7] D. Santel et al. [Belle Collaboration], Phys. Rev. D 93, 011101 (2016).

[8] R. Mizuk et al. [Belle Collaboration], Phys. Rev. Lett. 117, 142001 (2016).

[9] X. H. He et al. [Belle Collaboration], Phys. Rev. Lett. 113, 142001 (2014).

[10] Q. Huang, B. Wang, X. Liu, D. Y. Chen and T. Matsuki, Eur. Phys. J. C 77, 165 (2017). 